\title{
Dual-frequency Reflectarray Cells to Generate Closely Spaced Beams in Orthogonal Circular Polarization
}

\author{
Daniel Martínez de Rioja , Rafael Florencio , Jose A. Encinar ,Rafael R. Boix and Eduardo Carrasco
}

A dual-frequency reflectarray cell is proposed in this work to provide a progressive phase shift of opposite sign in each circular polarization (CP), by applying a variable rotation technique (VRT) [1]. The proposed reflectarray cell consists of two symmetrical arcs printed on the top surface and two orthogonal sets of parallel dipoles printed on two levels of a grounded dielectric substrate, see Figure 1. The arcs and dipoles are rotated independently to control the phase in $\mathrm{CP}$ at the lower $(19.7 \mathrm{GHz})$ and higher $(29.5 \mathrm{GHz})$ frequencies respectively. This reflectarray cell can be used to generate two adjacent beams in orthogonal $\mathrm{CP}$ per feed at transmission (Tx) and reception (Rx), in order to reduce the number of feeds and antennas required in current multi-spot satellites operating in Ka-band, as proposed in [1] for a single frequency band.

A home-made analysis routine based on the Method of Moments in the Spectral Domain (SD-MoM) and the local periodicity approach has been used for the analysis of the cell. This technique has been applied in previous works to analyze other reflectarray cells based on similar resonant elements, obtaining accurate results [2]. The length of the printed arcs has been adjusted to ensure a phase-shift of $180^{\circ}$ at $19.7 \mathrm{GHz}$ between the linear components in the rotated cell, which is the condition for VRT. On the other hand, the lengths of each set of dipoles can be varied to provide an additional phase-shift and to keep the required $180^{\circ}$ phase-shift between linear components. Assuming dielectrics $\left(\varepsilon_{r}=2.3\right)$ of thickness $1.524-\mathrm{mm}$ for the lower and $0.127 \mathrm{~mm}$ for the upper layers, the simulated phase of the co-polar components of reflection coefficients $\left(R_{X X}\right.$ and $\left.R_{Y Y}\right)$ at 29.5 $\mathrm{GHz}$ varies with the lengths of the dipoles in a range larger than $400^{\circ}$, as shown in Figure 1(b). These results show a good concordance between the curves obtained by CST Microwave Studio $(\mathbb{B})$ and the SD-MoM software. The phase variation produced by the dipoles can be used to properly shape the beam at $29.5 \mathrm{GHz}$. For example, in satellite antennas operating at Tx (19.2 -20.2 GHz) and Rx (29-30 GHz), the beam at Rx should be shaped to ensure the same beamwidth at Tx and Rx. It has been checked that the phases for both orthogonal CP's are almost independent for Tx and Rx and they are proportional to twice the rotation angle of arcs at Tx and twice the rotation angle of dipoles at Rx, respectively. Figure 1(c) shows the phase-shift at $19.7 \mathrm{GHz}$ for both CP components obtained by CST and SD-MoM, showing a good agreement.

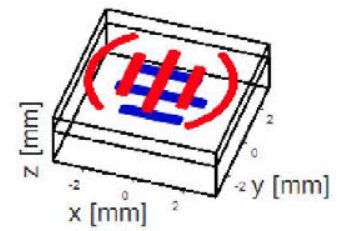

(a)

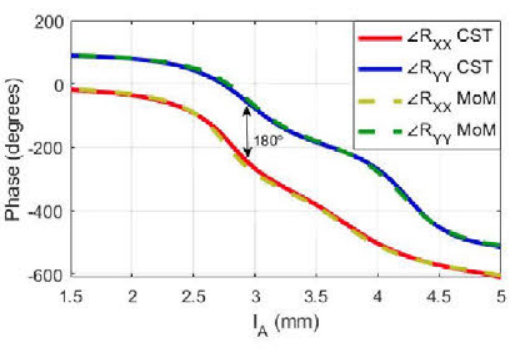

(b)

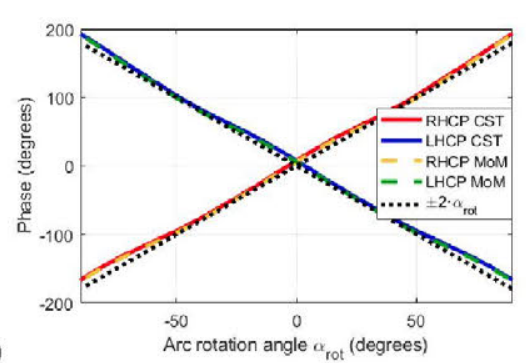

(c)

Figure 1. Reflectarray cells (a) and simulated phases: for linear compponents vs. lengths of central dipoles at $29.5 \mathrm{GHz}$ (b) and for CP components at $19.7 \mathrm{GHz}$ (c).

The proposed reflectarray cell could be used on a parabolic surface to generate two adjacent beams at Tx and $\mathrm{Rx}$ frequencies for each feed operating in dual-CP. This concept, applied to multi-spot satellites in Ka band, will allow a reduction in the number of feeds and antennas with respect to conventional reflectors.

This work has been supported by the Spanish Ministry of Economy and Competitiveness under project TEC2016-75103-C2-1-R and by the European Space Agency (ESA) under contract 4000117113/16/NL/AF.

1. M. Zhou and S. B. Sørensen, "Multi-spot beam reflectarrays for satellite telecommunication applications in Ka-band", 10th Eur. Conf. Antennas Propag. (EuCAP), Davos, 2016, pp.1-5.

2. R. Florencio, R. R. Boix, E. Carrasco, J. A. Encinar and V. Losada, "Efficient numerical tool for the analysis and design of reflectarrays based on cells with three parallel dipoles", Microw. Opt. Technol. Lett., vol. 55, no. 6, June 2013, pp. 1212- 1216. 